\title{
DPYD, TYMS and MTHFR Genes Polymorphism Frequencies in a Series of Turkish Colorectal Cancer Patients
}

\author{
Arsalan Amirfallah ${ }^{1,2,3}$, Gizem Calibasi Kocal ${ }^{3,4}$, Olcun Umit Unal ${ }^{5}$, Hulya Ellidokuz ${ }^{6,7}$, \\ Ilhan Oztop ${ }^{8}$ and Yasemin Basbinar $4,7,9, *$ (D)
}

1 Biomedical Center, Faculty of Medicine, University of Iceland, 101 Reykjavik, Iceland; arsalan.amirfallah@gmail.com

2 Cell Biology Unit, Department of Pathology, Landspitali University Hospital, 101 Reykjavik, Iceland

3 Department of Basic Oncology, Institute of Oncology, Dokuz Eylul University, 35350 Izmir, Turkey; gizem.calibasi@deu.edu.tr

4 Personalized Medicine and Pharmacogenomics/Genomics Research Centre-BIFAGEM, 3535 Izmir0, Turkey

5 Bozyaka Education and Research Hospital, Division of Medical Oncology, Department of Internal Medicine, Health Science University, 35170 Izmir, Turkey; drolcun@hotmail.com

6 Department of Preventive Oncology, Institute of Oncology, Dokuz Eylul University, 35350 Izmir, Turkey; hulya.ellidokuz@deu.edu.tr

7 Department of Medical Informatics and Biostatistics, Faculty of Medicine, Dokuz Eylul University, 35350 Izmir, Turkey

8 Department of Clinical Oncology, Faculty of Medicine, Dokuz Eylul University, 35350 Izmir, Turkey; ilhan.oztop@deu.edu.tr

9 Department of Translational Oncology, Institute of Oncology, Dokuz Eylul University, 35350 Izmir, Turkey

* Correspondence: yasemin.baskin@deu.edu.tr; Tel.: +90-232-412-58-90

Received: 19 October 2018; Accepted: 10 December 2018; Published: 13 December 2018

check for updates

\begin{abstract}
Fluoropyrimidine-based chemotherapy is extensively used for the treatment of solid cancers, including colorectal cancer. However, fluoropyrimidine-driven toxicities are a major problem in the management of the disease. The grade and type of the toxicities depend on demographic factors, but substantial inter-individual variation in fluoropyrimidine-related toxicity is partly explained by genetic factors. The aim of this study was to investigate the effect of dihydropyrimidine dehydrogenase (DPYD), thymidylate synthase (TYMS), and methylenetetrahydrofolate reductase (MTHFR) polymorphisms in colorectal cancer patients. Eighty-five patients who were administered fluoropyrimidine-based treatment were included in the study. The DPYD, TYMS and MTHFR polymorphisms were scanned by a next generation Sequenom MassARRAY. Fluoropyrimidine toxicities were observed in $92 \%$ of all patients. The following polymorphisms were detected: DPYD 85T $>C$ (29.4\% heterozygote mutants, 7.1\% homozygote mutants), DPYD IVS 14+1G>A (1.2\% heterozygote mutants), TYMS 1494del TTAAAG (38.4\% heterozygote mutants, 24.7\% homozygote mutants), MTHFR 677C >T ( $43.5 \%$ heterozygote mutants, $9.4 \%$ homozygote mutants) and MTHFR $1298 \mathrm{~A}>\mathrm{C}(8.2 \%$ heterozygote mutants, $2.4 \%$ homozygote mutants). A statistically significant association was demonstrated between MTHFR $677 \mathrm{C}>\mathrm{T}$ and fluoropyrimidine-related toxicity $(p$ value $=0.007)$. Furthermore, MTHFR 1298A $>\mathrm{C}$ was associated with hematopoietic toxicity $(p$ value $=0.008)$. MTHFR polymorphisms may be considered as related factors of fluoropyrimidine toxicity and may be useful as predictive biomarkers for the determination of the colorectal cancer patients who can receive the greatest benefit from fluoropyrimidine-based treatments.
\end{abstract}

Keywords: DPYD; TYMS and MTHFR genes; polymorphisms; pharmacogenetics; colorectal cancer 


\section{Introduction}

Combination chemotherapy regimens, including fluoropyrimidine-based chemotherapy, mainly with 5-fluorouracil (5-FU), have been a standard treatment for colorectal cancer for many years [1]. Dihydropyrimidine dehydrogenase (DPD), thymidylate synthase (TS) and methylenetetrahydrofolate reductase (MTHFR) are important enzymes for 5-FU metabolism. Over $80 \%$ of 5-FU is metabolized by DPD in the liver. The conversion of 5-FU to the inactive dihydrofluorouracil (DHFU) through DPD is the rate-limiting step of the 5-FU catabolism [2,3]. TS catalyzes the conversion of deoxyuridine monophosphate (dUMP) to deoxythymidine monophosphate (dTMP), which is critical for DNA replication. The active metabolite of 5-FU forms a ternary complex with TS and 5,10-methylenetetrahydrofolate (5,10-MTHF), and leads to the lack of dTMP. The absence of dTMP leads to reduced DNA synthesis, dUMP misincorporation into DNA, and DNA damage (double and single-strand breaks), followed by cell apoptosis [4,5]. MTHFR plays a role in the metabolism of folate. The substrate for MTHFR-5,10-MTHF-is used for the conversion of dUMP to dTMP by TS, whereas the product of 5-methyltetrahydrofolate is the methyl donor for the synthesis of methionine and $S$-adenosylmethionine in methylation reactions [6]. Studies have showed that 5-FU-based therapies prolong survival and reduce the risk of relapse. Clinically, colorectal cancer patients treated with combination regimes, including 5-FU, experience unavoidable treatment-related toxicities such as diarrhea, stomatitis, nausea, mucositis, myelosuppression, and hand-foot syndrome [1]. The grade and type of the toxicities depend on demographic factors such as sex, age, fluoropyrimidine application dose and mode of administration $[7,8]$. Substantial inter-individual variation in fluoropyrimidine-related toxicity is partly explained by genetic factors. The polymorphisms in the dihydropyrimidine dehydrogenase (DPYD), thymidylate synthase (TYMS), and methylenetetrahydrofolate reductase (MTHFR) genes have been reported as the reason for severe adverse reactions [9]. The aim of this study was to investigate the effect of DPYD, TYMS and MTHFR polymorphisms on the observed toxicities associated with fluoropyrimidine-based treatment in colorectal patients.

\section{Materials and Methods}

\subsection{Study Population and Setting}

Eighty-five colorectal cancer patients who were treated with fluoropyrimidine-based chemotherapy regimens between 2011 and 2013 at the Dokuz Eylul University Hospital were enrolled in this retrospective study. Informed consent was obtained from all patients. The study was based on routine blood material approved by the Non-Invasive Research Clinical Research Ethics Committee of Dokuz Eylul University School of Medicine (No: 2012/05-08). All patients had to fulfill the following inclusion criteria for the study: (i) diagnosed with a colorectal tumor and had fluoropyrimidine-based chemotherapy in Dokuz Eylul University Hospital, (ii) adequate hematological and cardiac status, (iii) sufficient biological material for genotyping analysis. Clinical data were obtained from standardized patient records.

\subsection{Demographic Data}

The characteristics of the 85 colorectal cancer patients treated with fluoropyrimidine-based agents are shown in Table 1. The mean age was 58.88 (range 20-81). The majority of the patients were men $(67.1 \%)$. The tumor location was predominantly the colon $(67.1 \%)$ and rectum $(32.9 \%)$. The histopathological type and the stage of the tumors were mostly observed as adenocarcinoma (91\%) and stage IV $(60 \%)$, respectively. The staging of the tumor was conducted when a patient was first diagnosed, before any treatment was given, according to the American Joint Committee on Cancer's (AJCC) Cancer Staging 6th edition 2002 TNM grading system [10]. 
Table 1. Patient characteristics of the study cohort.

\begin{tabular}{lcc}
\hline Demographic Details & & Patients $\%$ \\
\hline \multirow{2}{*}{ Gender $n(\%)$} & Male & 67.1 \\
& Female & 32.9 \\
\hline \multirow{2}{*}{ Age } & Range & $20-81$ \\
& Mean & 58.88 \\
\multirow{2}{*}{ Primary Tumor Site } & Colon & 67.1 \\
& Rectum & 32.9 \\
\hline \multirow{2}{*}{ Histopathological Type } & Adenocarcinoma & 91 \\
& Other & 9 \\
\hline \multirow{2}{*}{ Stage of Tumor } & I & 0 \\
& II & 9.4 \\
& III & 30.6 \\
\hline
\end{tabular}

\subsection{Toxicity Evaluation}

The toxicities associated with fluoropyrimidine-based treatment (such as gastrointestinal, hematopoietic, hair-skin toxicities and hand-foot syndrome) during the first two cycles of chemotherapy were evaluated at each clinic visit by anamnesis, physical examination, and the results of hematological, biochemical and urine tests. All adverse drug reactions and toxicities in the study group were recorded and graded according to the Common Terminology Criteria for Adverse Events v4.0 (CTCAE). Mucositis, ascites, colitis, diarrhea, dry mouth, gastritis, nausea and vomiting were grouped as gastrointestinal toxicity; anemia, febrile neutropenia and leukocytosis were grouped as hematopoietic toxicity; alopecia, dry skin, nail discoloration, nail loss, nail ridging, purpura, skin hyperpigmentation, skin hypopigmentation and skin ulceration were grouped as hair and skin toxicity; and hand-foot syndrome was placed in a discrete group. Grade 1 reflected a mild reaction, grade 2 a moderate reaction, grade 3 a reaction that was severe or medically significant but not immediately life-threatening, and grade 4 indicated a life-threatening adverse drug reaction.

\subsection{Genotyping}

Patients' peripheral blood samples were collected in EDTA-supplemented tubes. Samples were stored at $-80{ }^{\circ} \mathrm{C}$ until testing. Genomic DNA was extracted using the Invisorb Spin Blood Mini Kit (Invitek, Berlin, Germany). Single nucleotide polymorphism (SNP) genotyping of the DPYD, TYMS and MTHFR genes was performed with a MassARRAY platform (Sequenom Inc., San Diego, CA, USA), which relies on the natural molecular weight differences of DNA bases. This high-throughput platform combines the sensitivity of the polymerase chain reaction (PCR) and the accuracy of matrix-assisted laser desorption-ionization time-of-flight mass spectrometry (MALDI-TOF MS) [11]. PCR and iPLEX (Sequenom Inc., San Diego, CA, USA )single base extension primers for the multiplexed assays were designed with the MassARRAY ${ }^{\circledR}$ Designer software (Sequenom Inc.). Initial-locus-specific PCR reactions and locus-specific primer extension reactions (iPLEX assay) with oligonucleotide primers were performed to amplify specific polymorphic sites. MALDI-TOF mass spectrometry was used to distinguish the differences in the masses of allele-specific primer extension products for $D P Y D$ IVS 14+1G>A (DPYD*2A allele), DPYD 85T>C (DPYD*9A allele), DPYD 1679T >G/T (DPYD*13A allele), DPYD 2846A > T, TYMS $1494 \mathrm{ins/del,} \mathrm{MTHFR} \mathrm{677C}>$ T and MTHFR 1298A >C polymorphisms. The obtained data were analyzed using the MassARRAY Typer 4.0 Analyzer software (Sequenom Inc.).

\subsection{Statistical Analysis}

The Statistical Package for the Social Sciences (SPSS) (Version 21.0; SPSS, Inc., Chicago, IL, USA) program was used for statistical analysis. Gender, tumor location, histologic type of tumor, stage 
of tumor, observed toxicities, and frequency of polymorphisms defined as percentage of patients were analyzed. Toxicities were grouped by four classes, including gastrointestinal toxicity (diarrhea, mucositis, nausea), hematopoietic toxicity (myelosuppression), skin-hair toxicity (dry, cracking, peeling skin, hyperpigmentation, alopecia, hair thinning) and hand-foot syndrome. The Hardy-Weinberg equilibrium (HWE) of genotypes was analyzed by chi square test. The genotypes were discussed in relation to the previously published frequencies and Database of Single Nucleotide Polymorphisms (dbSNP) [12]. The chi- square test was used to determine the association of DPYD, TYMS and MTHFR polymorphisms with the existence of toxicity. A $p$ value $<0.05$ was considered as significant.

\section{Results}

\subsection{Patients' Toxicity Types and Grades}

Based on the Common Terminology Criteria for Adverse Events v4.0 (CTCAE), 91.8\% $(n=78)$ of the patients suffered from toxicities (Grade 1: 32.9\%, Grade 2: 22.4\%, Grade 3: 29.4, Grade 4: 7.1\%). According to the toxicity classification, hematopoietic toxicity (54.1\%) (Grade 1: 16.5\%, Grade 2: 9.4\%, Grade 3: 21.2\%, Grade 4: 7.1\%), hand-foot syndrome (21.2\%) (Grade 1: 9.4\%, Grade 2: 7.1\%, Grade 3: 4.7\%), gastrointestinal (12.9\%) (Grade 1: 5.9\%, Grade 2: 5.9\%, Grade 3: 1.2\%) and skin-hair toxicities (3.5\%) (Grade 1: 1.2\%, Grade 3: 2.4\%) were observed (Table 2).

Table 2. Major types of toxicity in patients receiving 5-fluorouracile-based chemotherapy in the first four cycles of treatment.

\begin{tabular}{ccccc}
\hline \multirow{2}{*}{ Toxicity Types } & \multicolumn{5}{c}{ Grade of Toxicities, \% } \\
\cline { 2 - 5 } & Grade $\mathbf{1}$ & Grade 2 & Grade 3 & Grade 4 \\
\hline Gastrointestinal toxicity & 5.9 & 5.9 & 1.2 & - \\
Hematopoietic toxicity & 16.5 & 9.4 & 21.2 & 7.1 \\
Hair and skin toxicity & 1.2 & - & 2.4 & - \\
Hand-foot Syndrome & 9.4 & 7.1 & 4.7 & - \\
\hline
\end{tabular}

\subsection{Patient Genotypes}

The polymorphism distribution is presented in Table 3. DPYD 85T $>\mathrm{C}$ (mutant allele frequency 0.22; polymorphism frequencies; $29.4 \%$ heterozygote mutants, $7.1 \%$ homozygote mutants), DPYD IVS $14+1 \mathrm{G}>\mathrm{A}$ (mutant allele frequency 0.01 ; polymorphism frequencies; $1.2 \%$ heterozygote mutants, $0 \%$ homozygote mutants), TYMS 1494 del TTAAAG (mutant allele frequency 0.44; polymorphism frequencies; $38.4 \%$ heterozygote mutants, $24.7 \%$ homozygote mutants), MTHFR 677C $>\mathrm{T}$ (mutant allele frequency 0.31 ; polymorphism frequencies; $43.5 \%$ heterozygote mutants, $9.4 \%$ homozygote mutants) and MTHFR 1298A >C (mutant allele frequency 0.09; polymorphism frequencies; $8.2 \%$ heterozygote mutants, 2.4\% homozygote mutants). Determined SNPs were in Hardy-Weinberg Equilibrium ( $p$ values were $0.955,0.208,0.049,0.895$ and 0.001 for DPYD IVS14+1G>A, DPYD 85T>C, TYMS 1494 del TTAAAG, MTHFR 677C $>$ T and MTHFR 1298A $>C$ respectively). The DPYD 1679T>G/T and DPYD $2846 \mathrm{~A}>\mathrm{T}$ polymorphisms were not detected in our group. 
Table 3. Genotyped polymorphisms and allelic frequencies of detected polymorphisms among all patients.

\begin{tabular}{|c|c|c|c|c|c|c|c|}
\hline \multirow{2}{*}{ Polymorphism } & \multirow{2}{*}{ SNP no } & \multirow{2}{*}{ Amino Acid Change } & \multicolumn{3}{|c|}{ Genotype Frequency \% } & \multicolumn{2}{|c|}{$\begin{array}{l}\text { Allelic } \\
\text { Frequency }\end{array}$} \\
\hline & & & $w t / w t^{1}$ & $w t /$ mut $^{2}$ & mut/mut & wt & mut \\
\hline $\begin{array}{c}D P Y D * 2 A \\
(\text { IVS14+1G>A) }\end{array}$ & rs3918290 & $\begin{array}{l}\text { Exon skipping- Exon } \\
14\end{array}$ & $\begin{array}{c}\text { GG } \\
(98.8)\end{array}$ & $\begin{array}{l}\text { AG } \\
(1.2)\end{array}$ & $\begin{array}{l}\text { AA } \\
(0)\end{array}$ & 0.99 & 0.01 \\
\hline $\begin{array}{l}D P Y D^{* 9} A \\
85 \mathrm{~T}>C\end{array}$ & rs1801265 & Cys29Arg- Exon 2 & $\begin{array}{c}\mathrm{TT} \\
(63.5)\end{array}$ & $\begin{array}{c}\text { TC } \\
(29.4)\end{array}$ & $\begin{array}{l}\mathrm{CC} \\
(7.1)\end{array}$ & 0.78 & 0.22 \\
\hline $\begin{array}{c}D P Y D * 13 \\
1679 \mathrm{~T}>\mathrm{G} / \mathrm{T}\end{array}$ & rs55886062 & I560S- Exon 13 & $\begin{array}{l}\mathrm{AA} \\
(100) \\
\end{array}$ & $\begin{array}{l}\mathrm{AC} \\
(0)\end{array}$ & $\begin{array}{l}\mathrm{CC} \\
(0) \\
\end{array}$ & 1 & 0 \\
\hline $\begin{array}{c}D P Y D \\
2846 \mathrm{~A}>\mathrm{T}\end{array}$ & rs67376798 & D949V- Exon 22 & $\begin{array}{l}\text { AA } \\
(100)\end{array}$ & $\begin{array}{l}\text { AT } \\
(0)\end{array}$ & $\begin{array}{l}\text { TT } \\
(0)\end{array}$ & 1 & 0 \\
\hline $\begin{array}{c}\text { TYMS } 1494 \\
\text { del TTAAAG }\end{array}$ & rs34489327 & $\begin{array}{c}6 \text { bp deletion-3'-UTR } \\
\text { region }\end{array}$ & $\begin{array}{c}(-;-) \\
(36.5)\end{array}$ & $\begin{array}{l}(-; \text { TTAAAG }) \\
\quad(38.8)\end{array}$ & $\begin{array}{c}\text { (TTAAAG;TTAAAG) } \\
(24.7)\end{array}$ & 0.56 & 0.44 \\
\hline $\begin{array}{l}\text { MTHFR } \\
677 \mathrm{C}>\mathrm{T}\end{array}$ & rs1801133 & A222V- Exon 4 & $\begin{array}{c}\text { CC } \\
(47.1) \\
\end{array}$ & $\begin{array}{c}\mathrm{CT} \\
(43.5) \\
\end{array}$ & $\begin{array}{c}\text { TT } \\
(9.4) \\
\end{array}$ & 0.69 & 0.31 \\
\hline $\begin{array}{c}\text { MTHFR } \\
1298 \mathrm{~A}>\mathrm{C}\end{array}$ & rs1801131 & E429A- Exon 7 & $\begin{array}{c}\text { AA } \\
(89.4)\end{array}$ & $\begin{array}{l}\mathrm{AC} \\
(8.2)\end{array}$ & $\begin{array}{l}\mathrm{CC} \\
(2.4)\end{array}$ & 0.91 & 0.09 \\
\hline
\end{tabular}

\subsection{Impact of Gene Polymorphisms on Toxicity}

At least one of four toxicity groups (gastrointestinal, hematopoietic, skin-hair toxicity and hand-foot syndrome) was observed in all patients with the MTHFR $677 \mathrm{C}>\mathrm{T}$ polymorphism (heterozygote/homozygote) ( $p$ value, 0.007) (Table 4). However, there was no association between MTHFR 677C $>$ T polymorphism (heterozygote/homozygote) and a specific toxicity group. The existence of the MTHFR 1298A >C polymorphism (heterozygote/homozygote) was associated with hematopoietic toxicity ( $p$ value, 0.008 ) (Table 5). Statistically significant associations could not be demonstrated between DPYD, TYMS polymorphisms and fluoropyrimidine-driven toxicities.

Table 4. Correlation of MTHFR 677C>T polymorphism with the presence of fluoropyrimidine-driven toxicities.

\begin{tabular}{cccccc}
\hline MTHFR 677C $>$ T & \multicolumn{2}{c}{ Toxicity $(n)$} & Risk Ratio & 95\% CI & $p$ Value \\
\hline \multicolumn{7}{c}{+} & - & & \\
\hline Het (CT) + Hom $(\mathrm{TT})$ & $45^{1}$ & 0 & 1.18 & $(1.03-1.34)$ & 0.007 \\
Wild Type (CC) & 34 & 6 & &
\end{tabular}

Table 5. Correlation of MTHFR 1298A >C polymorphism with presence of fluoropyrimidine-driven hematopoietic toxicity.

\begin{tabular}{cccccc}
\hline MTHFR 1298A $>$ C & Hematopoietic Toxicity $(\boldsymbol{n})$ & Risk Ratio & $\mathbf{9 5 \%}$ CI & $p$ Value \\
\hline Het (AC) + Hom (CC) & + & - & & \\
Wild Type (AA) & $9^{1}$ & 0 & \multirow{2}{*}{1.86} & $(1.50-2.32)$ & 0.008 \\
\hline
\end{tabular}

${ }^{1}$ heterozygous $(n=2)+$ Homozygous mutant $(n=7)$.

\section{Discussion}

The most widely used chemotherapy drugs in the treatment of colorectal cancer patients are 5-FU and capecitabine (pro-drug of 5-FU). Thymidylate synthase (TS), dihydropyrimidine dehydrogenase (DPD) and methylenetetrahydrofolate reductase (MTHFR) enzymes play important roles in the metabolic pathway of this chemotherapeutic [13-15]. In this study, common polymorphisms 
in the DPYD, TYMS and MTHFR genes that encode the DPD, TS and MTHFR enzymes, were investigated to understand their effects on fluoropyrimidine-related toxicity in Turkish colorectal cancer patients. Genomic DNA from eighty-five colorectal patients was analyzed for DPYD IVS14+1G $>$ A (DPYD*2A allele), DPYD 85T>C (DPYD*9A allele), DPYD 1679T>G/T (DPYD*13 allele), DPYD 2846A $>$ T, TYMS 1494 del TTAAAG, MTHFR $677 C>$ T and MTHFR 1298A $>$ C polymorphisms using a MassARRAY platform.

In the study group, DPYD IVS 14+1G>A (DPYD*2A allele), DPYD 85T>C (DPYD*9A allele), TYMS 1494 del TTAAAG, MTHFR 677C $>$ T and MTHFR 1298A $>$ C polymorphisms were detected; although DPYD 1679T>G/T (DPYD*13A allele) and DPYD 2846A > T polymorphisms were not. The distribution of polymorphisms and allelic frequencies are presented in Table 3.

The DPD enzyme functions as a rate-limiting enzyme in the catabolism of 5-FU. The DPD enzyme catalyzes the conversion of 5-FU into 5-fluoro-5, 6-dihydrouracil (5-FUH2), 5-FUH2, is then converted to fluoro-beta-ureidopropionate (FUPA) and subsequently to fluoro-beta-alanine (FBAL) by dihydropyrimidinease (DPYS) and beta-ureidopropionase (UPB1) enzymes, respectively (Figure 1). Single nucleotide polymorphisms in the DPYD gene are responsible for low levels of DPD enzyme. This insufficient production of DPD results in excess drug accumulation and toxicity due to the inefficient catabolism of the drug. Different DPYD polymorphisms and polymorphism-driven functional defects in this enzyme could lead to abnormal 5-FU metabolism and a wide variety of clinical manifestations. A number of studies have reported that patients with a DPYD mutated allele suffered from severe toxicity after fluoropyrimidine-based treatments. $D P Y D$ 85T $>C$ polymorphism caused the formation of a DPYD*9A allele with the conversion of Cys29Arg. In our study, the genotype frequency of the DPYD $85 \mathrm{~T}>\mathrm{C}$ polymorphism was $29.4 \%$ as a heterozygote mutation and $7.1 \%$ as a homozygote mutation. Although some groups have reported that the DPYD 85T>C polymorphism is predictive of fluoropyrimidine-related diarrhea, hand-foot syndrome and possibly ocular toxicity, no association was found between the DPYD 85T>C polymorphism and toxicity in our group [16,17].

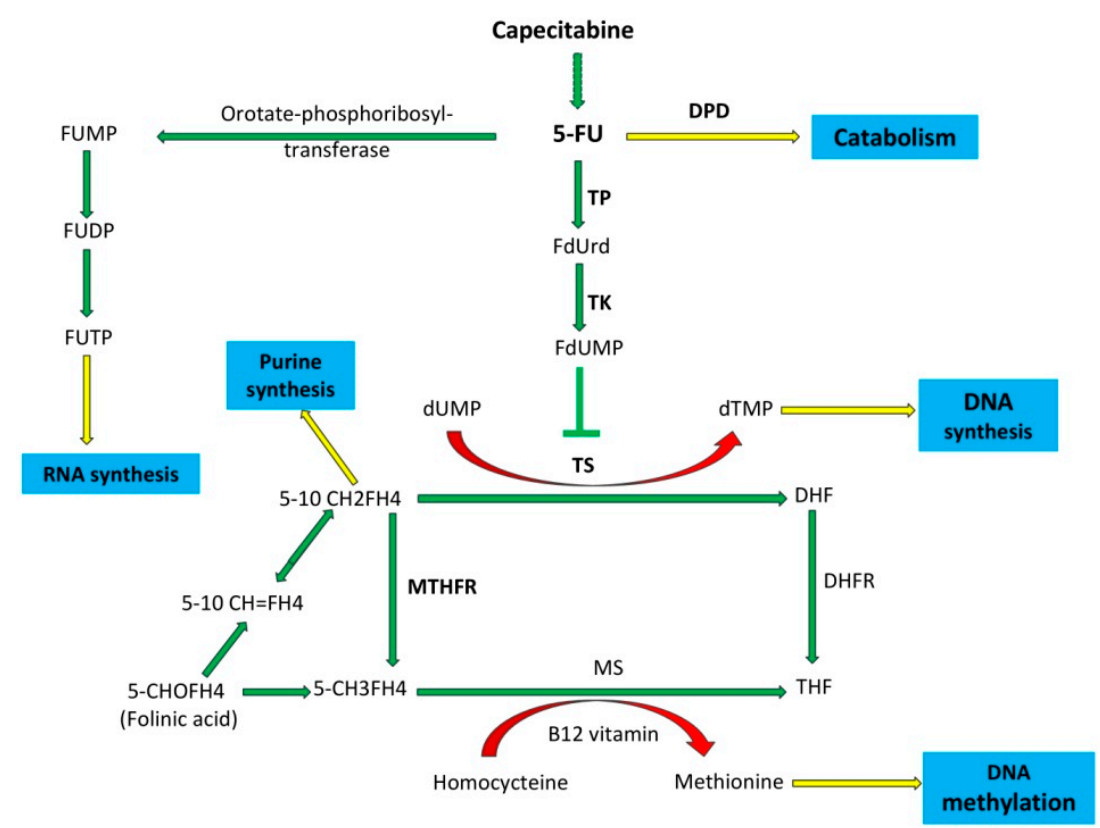

Figure 1. Metabolism pathway of capecitabine and 5-fluorouracil (5-FU). Abbreviations: 5-fluoro uracil (5-FU), Thymidine phosphorylase (TP), 5-fluorodeoxyuridine (FdUrd), thymidine kinase (TK), 5-10 methylene-tetrahydrofolate (5-10 CH2FH4), dihydrofolate (DHF), 5-methyltetrahydrofolate (5-CH3FH4), methionine synthase (MS), tetrahydrofolate (THF), dihydrofolate reductase (DHFR), 5-formyltetrahydrofolate (folinic-acid) (5-CHOFH4), and 5-10 methenyltetrahydrofolate (5-10 $\mathrm{CH}=\mathrm{FH} 4)$. 
The DPYD IVS14+1G>A splice-site transition results in a 165-bp deletion (corresponding to exon 14) in the DPYD mRNA and forms the DPYD*2A allele. The catalytic activity of the new truncated protein product is extremely low. The polymorphism analysis of the frequency showed that the IVS14+1G $>$ A polymorphism was the most common among the cancer patients suffering from severe 5-FU-associated toxicity. Several reports have been published regarding the IVS14+1G>A splice-site transition frequency in subjects of different ethnic backgrounds and appears most commonly in Caucasian people. However, in our group, this polymorphism was the second most common, with $1.2 \%$, among the DPYD polymorphisms [13,15].

DPYD 1679T $>$ G/T and DPYD 2846A $>$ T have consistently been associated with fluoropyrimidine toxicity. DPYD 1679T>G substitution results in the DPYD*13 allele due to the the change in I560S, and DPYD 2846A $>$ T causes D949V to change. Both of them have been reported to be associated with low enzyme activity but have been observed as rare polymorphisms. In this study, the DPYD 1679T $>\mathrm{G} / \mathrm{T}$ and DPYD 2846A $>\mathrm{T}$ polymorphisms were not detected $[18,19]$.

In this report, the distributions and allele frequencies of the various DPYD polymorphisms (IVS14+1G >A, 85T $>C, 1679 \mathrm{~T}>\mathrm{G} / \mathrm{T}, 2846 \mathrm{~A}>\mathrm{T}$ ) were found to be similar to previously published reports. Our results were similar to Caucasian/European-based studies on DPYD 85T $>\mathrm{C}$ and $D P Y D$ IVS14+1G>A polymorphisms. However, for DPYD 1679T $>$ G/T and DPYD 2846A $>\mathrm{T}$ polymorphisms, our results were compatible with Asian-based studies. The allelic frequencies of all DPYD polymorphisms in this study were similar to global minor allelic frequency (MAF) data from dbSNP [15]. In contrast to the similarity of the genetic distributions, a statistically significant association could not be demonstrated between DPYD polymorphisms and fluoropyrimidine toxicities. Ethnic variations can change the clinical presentation of the disease and the pharmacokinetic/dynamic processes of the drugs. There are no adequately published data from a large Turkish population that investigate this in this respect. For this reason, large population-based studies are urgently needed to successfully manage the disease. Various enzymes (e.g., cytidine deaminase or CDA) and different polymorphisms also have roles in 5-FU metabolism [20]. These different polymorphisms should also be checked for their association with toxicity.

The primary mechanism of action of 5-FU is TS inhibition with fluorodeoxyuridine monophosphate (FdUMP), an active metabolite of 5-FU. FdUMP, TS and 5-10-methylenetrahydrofolate (MTHF) form an inactive triple complex (Supplementary Figure S1). This inhibition leads to the accumulation of deoxy-uridine-monophospate (dUMP) and the depletion of deoxy-thymidine-monophosphate (dTMP), which lead to the inhibition of DNA synthesis. The 5-FU can also inhibit RNA synthesis in a pathway that involves 5-fluorouridine monophosphate (FUMP) and subsequent conversion to 5-fluorouridine triphosphate (FUTP) via 5-fluorouridine diphosphate (FUDP). The optimal formation of FdUMP/TS/MTHF inactive triple complex requires a high MTHF level regulated by the methylenetetrahydrofolate reductase enzyme (MTHFR) on the catabolism process of 5-FU. Therefore, both TS and MTHFR activity are thought to be the determining factor for the 5-FU clinical response [4].

The presence of TYMS 1494 del TTAAAG at the 3'-UTR of gene (an insertion/deletion of a 6-bp sequence) is known to influence TS expression by leading to the formation of a less stable mRNA that will be more susceptible to degradation and have a decreased TS expression. TYMS 1494 del TTAAAG has been associated with both fluoropyrimidine-related toxicity and increased clinical responses [20]. Moreover, in most of the studies that were conducted with Caucasian (German, French, and Italian) or Asian (Chinese, Japanese) populations (52-60\%), no significant association was found between the TYMS 1494 del TTAAAG polymorphism and fluoropyrimidine toxicity [21]. Although the TYMS 1494 del TTAAAG polymorphism was detected in $63.1 \%$ of all patients ( $38.4 \%$ heterozygote mutants, $24.7 \%$ homozygote mutants), a statistically significant association could not be demonstrated between the TYMS 1494 del TTAAAG polymorphism and the 5-FU toxicities. The potential importance of TYMS polymorphisms on fluoropyrimidine toxicity is still controversial. For accurate and decisive clinical results regarding TYMS, further systematic studies with large patient populations are required. 
MTHFR is another key enzyme for 5-FU metabolism. It catalyzes the conversion of 5,10 methylenetetrahydrofolate (5,10-MTHF) to 5-methylenetetrahydrofolate (5-MTHF) [22]. Despite finding more than 60 germline polymorphisms in MTHFR [23], only MTHFR 677C > T, MTHFR 1298A>C, which are nonsynonymous SNPs, reduce MTHFR enzyme activity [24].

In our group, the MTHFR $677 \mathrm{C}>\mathrm{T}$ frequency $(43.5 \%$ heterozygote mutants, $9.4 \%$ homozygote mutants) was similar to the Caucasian-based studies, and it is evident that this polymorphism is not common in Middle Eastern or African populations (Supplementary Table S1) [25]. The allelic frequency of MTHFR $677 \mathrm{C}>\mathrm{T}$ was determined to be 0.31 and was close to the global minor allelic frequency data from dbSNP [12].

The MTHFR 1298A>C polymorphism was quite rare in our group $(8.2 \%$ heterozygote mutants, $2.4 \%$ homozygote mutants). The frequency of this polymorphism is higher in Caucasian, Asian and African-based populations than in our study (Supplementary Table S1). The frequency of both polymorphisms varies significantly between regions and ethnic groups [22,25]. The allelic frequency of the MTHFR $1298 \mathrm{~A}>\mathrm{C}$ polymorphism was determined to be 0.09 , which is not even close to the global minor allelic frequency data (0.25) for dbSNP [12].

Although the MTHFR enzyme, which was involved in the 5-FU metabolic pathway, has been studied by several groups, the presented results have been controversial regarding the role of the MTHFR $677 C>$ T and MTHFR 1298 A $>$ C polymorphisms on fluoropyrimidine toxicity in colorectal cancer patients [7].

In our study, a statistically significant association was demonstrated between the MTHFR 677C >T polymorphism (heterozygous/homozygous) and fluoropyrimidine toxicity ( $p$ value 0.009 ) (Table 3 ). Our results were compatible with the literature. Afzal et al. and Toffili et al. reported that the MTHFR $677 \mathrm{C}>\mathrm{T}$ polymorphism was associated with fluoropyrimidine-related toxicity in colorectal and breast cancer patients, respectively, without claiming a specific toxicity type $[5,26]$. Lu et al. demonstrated an association between the MTHFR $677 \mathrm{C}>\mathrm{T}$ polymorphism and a higher frequency of nausea and vomiting in gastric cancer patients [27]. Noor et al. found a significant correlation of the MTHFR C677T polymorphism with increased tumor response to 5-FU and developing grade 3 or 4 neutropenia, diarrhea, and mucositis in colorectal cancer patients [28]. In contrast to these reports and our results, Capitain et al. found no significant association in MTHFR 677C $>$ T mutant patients in terms of 5-FU toxicity in patients with advanced colorectal cancer [29].

We found an association between the MTHFR 1298A>C polymorphism and hematopoietic toxicity. In the literature, the same contradicting results have been shown for the MTHFR 1298A>C polymorphism. Some researchers, such as Afzal et al., have reported no association between the MTHFR 1298A $>$ C polymorphism and toxicity [5]. However, several researchers have reported that the MTHFR 1298A >C polymorphism had importance for the prediction of toxicity or the type of toxicity. Thomas et al. showed that MTHFR 1298A $>$ C polymorphism is predictive of diarrhea or mucositis upon fluoropyrimidine administration as a single agent in rectal cancer patients [4]. Loganayagam et al. reported that both the MTHFR 677C $>\mathrm{T}$ and MTHFR 1298A $>\mathrm{C}$ polymorphisms were associated with hand-foot syndrome in gastrointestinal and colorectal patients [20]. Diarrhea, mucositis and hand-foot syndrome were also observed as fluoropyrimidine-related toxicities in the presence of the MTHFR 1298A $>$ C polymorphism. However, there have been no reports of hematopoietic toxicity in the presence of the MTHFR 1298A >C polymorphism in colorectal patients [5]. In our study, the patient group who used capecitabine was small. Therefore, we could not carry out further statistical analysis in regard to capecitabine. The same analysis should be made with a larger population of patients using capecitabine.

The MTHFR $677 \mathrm{C}>\mathrm{T}$ polymorphism shows a wide regional and ethnic diversity, with a genotype frequency from $12.6 \%$ to $44.5 \%$ for heterozygote mutations and from $0 \%$ to $13.2 \%$ for homozygote mutations. The frequencies of the homozygote (TT) or heterozygote (CT) genotypes among Caucasian/European populations are higher than in Asian, Middle Eastern and African populations. In this Turkish population-based study, the frequency results are close to those for 
Caucasians/Europeans. On the other hand, the MTHFR 1298A $>$ C polymorphism does not present as much population diversity. Its frequency is more uniform within the studied groups. Table 4 shows the frequencies of polymorphisms with the heterozygous and homozygous genotypes, according to different ethnic populations.

Although 5-FU metabolism-related polymorphisms have been studied by several groups, the presented results have been controversial regarding the effects of the polymorphisms on fluoropyrimidine toxicity in colorectal cancer patients.

The study groups generally included mixed ethnic populations, and ethnic variation affected the frequency and type of genetic mutations. Pharmacogenetic studies require a certain level of homogeneity in the selection of patients and therapies.

The weakness of this study was the limited number of patients. The association of the MTHFR polymorphisms used in this study with fluoropyrimidine-based chemotherapy-related toxicities in colorectal cancer patients needs to be confirmed in larger prospective study populations with a uniform chemotherapy regimen.

\section{Conclusions}

Fluoropyrimidine-based chemotherapeutic agents, 5-FU or capecitabine, have remained the mainstay of chemotherapeutic regimens for colorectal and gastrointestinal cancers in both metastatic and adjuvant settings. Therefore, it is important to identify decisive parameters that may help in the prognostic or predictive assessment of disease and drug toxicity. A drug response is a complex trait that involves many proteins. From a biological point of view, it is expected that different metabolic routes compete and that the effect of a polymorphism on a drug metabolism pathway can be altered by other polymorphisms [7]. Investigation into the role of polymorphisms influencing the fluoropyrimidine metabolism appears to be a promising area for further research.

Although our study population was small, according to our results, MTHFR $677 C>T$ can be used for the prediction of fluoropyrimidine-based chemotherapy toxicities and MTHFR 1298A>C polymorphisms for the prediction of hematopoietic toxicities in colorectal cancer patients.

All of our findings and the supporting or opposing reports from various groups need to be validated in larger population-based cohorts, and the results should be compared with the toxicities of both single agent-based or combination-based fluoropyrimidine therapies.

Supplementary Materials: The following are available online at http:/ / www.mdpi.com/2075-4426/8/4/45/s1, Figure S1: Catabolism of capecitabine and 5-fluorouracil (5-FU), Table S1: Genotype frequencies of MTHFR 677C $>\mathrm{T}$ and 1298A $>$ C polymorphisms in various ethnic groups including our study.

Author Contributions: A.A. methodology, validation, software, writing-original draft preparation, G.C.K. performing of experiments, data analysis, manuscript preparation, O.U.U. investigation, H.E. formal analysis, data curation, I.O. investigation, Y.B. project administration, funding acquisition, writing-review and editing.

Funding: This work was supported by Dokuz Eylul University Research Foundation [Grant number: 2013.KB.SAG.036].

Conflicts of Interest: The authors declare no conflict of interest.

\section{References}

1. Cremolini, C.; Del Re, M.; Antoniotti, C.; Lonardi, S.; Bergamo, F.; Loupakis, F.; Borelli, B.; Marmorino, F.; Citi, V.; Cortesi, E.; et al. DPYD and UGT1A1 genotyping to predict adverse events during first-line FOLFIRI or FOLFOXIRI plus bevacizumab in metastatic colorectal cancer. Oncotarget 2018, 9, 7859-7866. [CrossRef] [PubMed]

2. Amstutz, U.; Froehlich, T.K.; Largiader, C.R. Dihydropyrimidine dehydrogenase gene as a major predictor of severe 5-fluorouracil toxicity. Pharmacogenomics 2011, 12, 1321-1336. [CrossRef] [PubMed]

3. Ciccolini, J.; Gross, E.; Dahan, L.; Lacarelle, B.; Mercier, C. Routine dihydropyrimidine dehydrogenase testing for anticipating 5-fluorouracil-related severe toxicities: Hype or hope? Clin. Colorectal Cancer 2010, 9, $224-228$. [CrossRef] 
4. Thomas, F.; Motsinger-Reif, A.A.; Hoskins, J.M.; Dvorak, A.; Roy, S.; Alyasiri, A.; Myerson, R.J.; Fleshman, J.W.; Tan, B.R.; McLeod, H.L. Methylenetetrahydrofolate reductase genetic polymorphisms and toxicity to 5-FU-based chemoradiation in rectal cancer. Br. J. Cancer 2011, 105, 1654-1662. [CrossRef]

5. Afzal, S.; Jensen, S.A.; Vainer, B.; Vogel, U.; Matsen, J.P.; Sorensen, J.B.; Andersen, P.K.; Poulsen, H.E. MTHFR polymorphisms and 5-FU-based adjuvant chemotherapy in colorectal cancer. Ann. Oncol. 2009, 20, 1660-1666. [CrossRef] [PubMed]

6. Cohen, V.; Panet-Raymond, V.; Sabbaghian, N.; Morin, I.; Batist, G.; Rozen, R. Methylenetetrahydrofolate reductase polymorphism in advanced colorectal cancer: A novel genomic predictor of clinical response to fluoropyrimidine-based chemotherapy. Clin. Cancer Res. 2003, 9, 1611-1615. [PubMed]

7. Afzal, S.; Gusella, M.; Vainer, B.; Vogel, U.B.; Andersen, J.T.; Broedbaek, K.; Petersen, M.; Jimenez-Solem, E.; Bertolaso, L.; Barile, C.; et al. Combinations of polymorphisms in genes involved in the 5-Fluorouracil metabolism pathway are associated with gastrointestinal toxicity in chemotherapy-treated colorectal cancer patients. Clin. Cancer Res. 2011, 17, 3822-3829. [CrossRef] [PubMed]

8. Schwab, M.; Zanger, U.M.; Marx, C.; Schaeffeler, E.; Klein, K.; Dippon, J.; Kerb, R.; Blievernicht, J.; Fischer, J.; Hofmann, U.; et al. Role of genetic and nongenetic factors for fluorouracil treatment-related severe toxicity: A prospective clinical trial by the German 5-FU Toxicity Study Group. J. Clin. Oncol. 2008, 26, 2131-2138. [CrossRef] [PubMed]

9. Houtsma, D.; Guchelaar, H.J.; Gelderblom, H. Pharmacogenetics in oncology: A promising field. Curr. Pharm. Des. 2010, 16, 155-163. [CrossRef] [PubMed]

10. Greene, F.L.; Page, D.L.; Fleming, I.D.; Fritz, A.; Balch, C.M.; Haller, D.G.; Morrow, M. (Eds.) Cancer Staging Manual. In American Joint Committee on Cancer (AJCC); Digestive System; Lippincott Raven Publishers: Philadelphia, PA, USA, 2002; pp. 113-124.

11. Bradic, M.; Costa, J.; Chelo, I.M. Genotyping with Sequenom. Meth. Mol. Biol. 2011, 772, 193-210. [CrossRef]

12. Sherry, S.T.; Ward, M.-H.; Kholodov, M.; Baker, J.; Smigielski, E.M.; Sirotkin, K. dbSNP: The NCBI database of genetic variation. Nucleic Acids Res. 2001, 29, 308-311. [CrossRef] [PubMed]

13. Van Kuilenburg, A.B.; De Abreu, R.A.; van Gennip, A.H. Pharmacogenetic and clinical aspects of dihydropyrimidine dehydrogenase deficiency. Ann. Clin. Biochem. 2003, 40, 41-45. [CrossRef] [PubMed]

14. Maitland, M.L.; Vasisht, K.; Ratain, M.J. TPMT, UGT1A1 and DPYD: Genotyping to ensure safer cancer therapy? Trends Pharmacol. Sci. 2006, 27, 432-437. [CrossRef] [PubMed]

15. Sulzyc-Bielicka, V.; Binczak-Kuleta, A.; Pioch, W.; Kladny, J.; Gziut, K.; Bielicki, D.; Ciechanowicz, A. 5-Fluorouracil toxicity-attributable IVS14 + 1G > A mutation of the dihydropyrimidine dehydrogenase gene in Polish colorectal cancer patients. Pharmacol. Rep. 2008, 60, 238-242. [PubMed]

16. Mattison, L.K.; Soong, R.; Diasio, R.B. Implications of dihydropyrimidine dehydrogenase on 5-fluorouracil pharmacogenetics and pharmacogenomics. Pharmacogenomics 2002, 3, 485-492. [CrossRef] [PubMed]

17. Baskin, Y.; Amirfallah, A.; Unal, O.U.; Calibasi, G.; Oztop, I. Dihydropyrimidine dehydrogenase 85T $>$ C mutation is associated with ocular toxicity of 5-fluorouracil: A case report. Am. J. Ther. 2015, 22, e36-e39. [CrossRef]

18. Seck, K.; Riemer, S.; Kates, R.; Ullrich, T.; Lutz, V.; Harbeck, N.; Schmitt, M.; Kiechle, M.; Diasio, R.; Gross, E. Analysis of the DPYD gene implicated in 5-fluorouracil catabolism in a cohort of Caucasian individuals. Clin. Cancer Res. 2005, 11, 5886-5892. [CrossRef]

19. He, Y.F.; Wei, W.; Zhang, X.; Li, Y.H.; Li, S.; Wang, F.H.; Lin, X.B.; Li, Z.M.; Zhang, D.S.; Huang, H.Q.; et al. Analysis of the DPYD gene implicated in 5-fluorouracil catabolism in Chinese cancer patients. J. Clin. Pharm. Ther. 2008, 33, 307-314. [CrossRef]

20. Loganayagam, A.; Arenas Hernandez, M.; Corrigan, A.; Fairbanks, L.; Lewis, C.M.; Harper, P.; Maisey, N.; Ross, P.; Sanderson, J.D.; Marinaki, A.M. Pharmacogenetic variants in the DPYD, TYMS, CDA and MTHFR genes are clinically significant predictors of fluoropyrimidine toxicity. Br. J. Cancer 2013, 108, 2505-2515. [CrossRef]

21. Lima, A.; Azevedo, R.; Sousa, H.; Seabra, V.; Medeiros, R. Current approaches for TYMS polymorphisms and their importance in molecular epidemiology and pharmacogenetics. Pharmacogenomics 2013, 14, 1337-1351. [CrossRef]

22. De Mattia, E.; Toffoli, G. C677T and A1298C MTHFR polymorphisms, a challenge for antifolate and fluoropyrimidine-based therapy personalisation. Eur. J. Cancer 2009, 45, 1333-1351. [CrossRef] [PubMed] 
23. Martin, Y.N.; Salavaggione, O.E.; Eckloff, B.W.; Wieben, E.D.; Schaid, D.J.; Weinshilboum, R.M. Human methylenetetrahydrofolate reductase pharmacogenomics: Gene resequencing and functional genomics. Pharmacogenet. Genom. 2006, 16, 265-277. [CrossRef]

24. Frosst, P.; Blom, H.J.; Milos, R.; Goyette, P.; Sheppard, C.A.; Matthews, R.G.; Boers, G.J.; den Heijer, M.; Kluijtmans, L.A.; van den Heuvel, L.P.; et al. A candidate genetic risk factor for vascular disease: A common mutation in methylenetetrahydrofolate reductase. Nat. Genet. 1995, 10, 111-113. [CrossRef]

25. Bu, R.; Gutierrez, M.I.; Al-Rasheed, M.; Belgaumi, A.; Bhatia, K. Variable drug metabolism genes in Arab population. Pharmacogenom. J. 2004, 4, 260-266. [CrossRef] [PubMed]

26. Toffoli, G.; Veronesi, A.; Boiocchi, M.; Crivellari, D. MTHFR gene polymorphism and severe toxicity during adjuvant treatment of early breast cancer with cyclophosphamide, methotrexate, and fluorouracil (CMF). Ann. Oncol. 2000, 11, 373-374. [CrossRef] [PubMed]

27. Lu, J.W.; Gao, C.M.; Wu, J.Z.; Sun, X.F.; Wang, L.; Feng, J.F. Relationship of methylenetetrahydrofolate reductase C677T polymorphism and chemosensitivity to 5-fluorouracil in gastric carcinoma. Ai Zheng = Aizheng = Chin. J. Cancer 2004, 23, 958-962. (In Chinese)

28. Nahid, N.A.; Apu, M.N.H.; Islam, M.R.; Shabnaz, S.; Chowdhury, S.M.; Ahmed, M.U.; Nahar, Z.; Islam, M.S.; Islam, M.S.; Hasnat, A. DPYD*2A and MTHFR C677T predict toxicity and efficacy, respectively, in patients on chemotherapy with 5-fluorouracil for colorectal cancer. Cancer Chemother. Pharmacol. 2018, 81, 119-129. [CrossRef]

29. Capitain, O.; Boisdron-Celle, M.; Poirier, A.L.; Abadie-Lacourtoisie, S.; Morel, A.; Gamelin, E. The influence of fluorouracil outcome parameters on tolerance and efficacy in patients with advanced colorectal cancer. Pharmacogenom. J. 2008, 8, 256-267. [CrossRef]

(C) 2018 by the authors. Licensee MDPI, Basel, Switzerland. This article is an open access article distributed under the terms and conditions of the Creative Commons Attribution (CC BY) license (http:/ / creativecommons.org/licenses/by/4.0/). 